\title{
Fitness and life-history traits of the two major mitochondrial DNA haplotypes of Drosophila subobscura
}

\author{
JS Christie ${ }^{1}$, JA Castro ${ }^{1}$, P Oliver ${ }^{1}$, A Picornell ${ }^{1}$, MM Ramon $^{1}$ and A Moya ${ }^{2}$ \\ ${ }^{1}$ Laboratori de Genètica, Departament de Biologia, Facultat de Ciències, Edifici Guillem Colom, Universitat de les Illes Balears, Campus \\ de la UIB, Palma de Mallorca, Balears 07122, Spain; 'Institut 'Cavanilles' de Biodiversitat i Biologia Evolutiva i Departament de \\ Genètica, Universitat de València, Burjassot, València, Spain
}

\begin{abstract}
Mitochondrial DNA restriction site analyses on natural populations of Drosophila subobscura have proved the existence of two common, coexisting haplotypes (I and II), as well as a set of less frequent ones derived from them. To explain this distribution, experiments to date point practically to all possible genetic mechanisms being involved in the changes of gene frequencies (cytonuclear coadaptation, direct natural selection on mtDNA and genetic drift). In an attempt to find differences that help to understand the dynamics of these haplotypes and to detect the effect of selection, we measured certain fitness components and
\end{abstract}

life-history traits (egg-larva and larva-adult viabilities and developmental times, longevity, resistance to desiccation and optimal density) of the two main haplotypes I and II when maintained in laboratory population cages. As a general trend, haplotype II showed a higher net fitness than haplotype I, which explains the superiority of haplotype II over haplotype I in experimental populations but not their coexistence in nature, where additional factors must be considered.

Heredity (2004) 93, 371-378. doi:10.1038/sj.hdy.6800513

Published online 7 July 2004

Keywords: fitness components; natural selection; cytonuclear interactions; mtDNA haplotypes; Drosophila subobscura

\section{Introduction}

It is a constant observation in the Old World that Drosophila subobscura shows widespread geographical homogeneity with a high prevalence of two main and coexisting mitochondrial DNA (mtDNA) haplotypes at the level of restriction site analyses (named I and II), as well as a set of less common haplotypes, derived from these two, which appear at much lower frequencies (Latorre et al, 1992; García-Martínez et al, 1998; Castro et al, 1999; Oliver et al, 2002). Studies on populations colonizing the New World have given similar results (Rozas et al, 1990). The unique exception to date has been detected on the Canary Islands, where an endemic haplotype (named VIII) is predominant on some islands (Pinto et al, 1997).

In recent years, we have studied the dynamics of these haplotypes, trying to answer the questions as to why the two main haplotypes are approximately equally frequent in nature and which evolutionary forces are implicated. Moya et al (1993) studied the degree of differentiation of the two haplotypes at the nucleotide level. A total of $2377 \mathrm{bp}$ from six mtDNA functional regions, representing $15 \%$ of the mtDNA genome, were sequenced per haplotype. Only three differences were found and they

Correspondence: JA Castro, Laboratori de Genètica, Departament de Biologia, Facultat de Ciències, Edifici Guillem Colom, Universitat de les Illes Balears, Campus de la UIB, Palma de Mallorca, Balears 07122, Spain. E-mail: jose.castro@uib.es

Received 23 October 2003; accepted 1 April 2004; published online 7 July 2004 proved to be silent changes at the protein level, one of which corresponded to the HaeIII restriction site, located in the ND5 gene, which distinguishes the haplotypes. On the basis of these results, as well as others involving the geographical distribution, they considered haplotypes I and II to be phenotypically equivalent, although it is possible (and even probable) that further sequencing could reveal significant differences between the two mtDNAs in the remaining 85\% (Moya et al, 1993). In fact, when these haplotypes were put together at different frequencies in population cages, haplotype II always displaced haplotype I (García-Martínez et al, 1998). Likewise, in another study, when haplotypes I and VIII were put together to compete in population cages at different densities and nuclear backgrounds, the mtDNA haplotype that reached fixation was the one placed within its own nuclear background, thus indicating the importance of nuclear-mtDNA coadaptation (Fos et al, 1990). More recently, Castro et al (1999) and Oliver et al (2002) proved the existence of transient cytonuclear disequilibria, with nuclear allozyme loci and chromosomal arrangements. In summary, different studies to date have pointed practically to all the populational mechanisms that can change gene frequencies (cytonuclear coadaptation, direct natural selection on mtDNA and random genetic drift) as being forces potentially acting on the mtDNA haplotypes.

The present research falls within the series of studies that many groups have been carrying out in recent years, on the populational forces that are acting on the mtDNA. In the literature, studies concerning the population genetic dynamics of mtDNA have often, although not 
always, rejected neutral patterns, mainly in humans and their associated commensal taxa (flies and mice) (see, Gerber et al, 2001, for a recent revision). Similarly, studies on other species of Drosophila have also observed nonneutral behaviour of mtDNA variants (Hutter and Rand, 1995) and have provided evidence that maintenance of mtDNA variability could be mediated through cytonuclear interactions (Clark and Lyckegaard, 1988; MacRae and Anderson, 1988; but see Singh and Hale, 1990, for an alternative view). Rand et al (2001) recently showed that nuclear-cytoplasmic polymorphisms could also be maintained by interaction between X-chromosomes and cytoplasm.

In the present work, research was focused on fitness components and life-history traits as components of natural selection. These components have been extensively reported in the literature for several organisms. In Drosophila, the adaptation to novel environments involving high-density conditions has been studied (Mueller et al, 1993), as well as senescence (Rose, 1984), starvation (Rose et al, 1992), temperature (Huey et al, 1991), fecundity, longevity and developmental time (Matos et al, 2000), and population divergence (Kennington et al, 2001) among others.

Therefore, the purpose of this work was the detection of the differential action of natural selection on flies with these mtDNA haplotypes. The study was a continuation of the work we have been undertaking on the forces acting on the populational dynamics of the two haplotypes (I and II). Thus, we studied the egg-larva and larva-adult viabilities and developmental times, longevity and the resistance to desiccation. In addition, we also studied the optimal density (OD) (density at which the food medium yields the highest number of adult individuals) to measure the way in which the medium is exploited (Wallace, 1981; Castro et al, 1985). Other fitness components, such as mating pattern and female fertility, which are especially important in mtDNA dynamics due to maternal inheritance, were studied in flies from population cages (the same used in the present paper) and in a recently collected lines from a natural population (Castro et al, 2003).

\section{Materials and methods}

\section{Haplotype populations}

Two different populational cages were founded, one with haplotype I and another with haplotype II. The cage with haplotype I was created with 71 isofemale lines and the cage with haplotype II with 98 . The isofemale lines were collected in a pine forest near Calvià (Majorca, Spain) in the spring of 1997, and the mtDNA haplotypes determined as indicated in Castro et al (1999). Each isoline contributed approximately five males and five females. They were maintained in the laboratory at $19^{\circ} \mathrm{C}, 70 \%$ relative humidity, a 1:1 day-night cycle and with 12 jars containing corn-meal food. The mean number of individuals was approximately 1500 per generation. From these cages, two samples, one with 18 haplotype I isofemales and another with 21 haplotype II isofemales, were obtained in order to establish separate populations for the experiments. When these samples were taken, the cages had passed nine discrete generations. The mtDNA haplotypes of these isofemales were checked through the offspring by a simple mtDNA analysis (Castro et al, 1999). The use of 18 and of 21 isofemale lines, respectively, should be enough to capture $95 \%$ of the variability in nuclear alleles that is present in the cages (Hedrick, 2000, p. 240). These two new populations were maintained by serial transfer in five $500 \mathrm{ml}$ bottles, during the time that the experiments were being performed.

\section{Presence of Wolbachia}

To exclude an incompatibility system in D. subobscura, promoted by the presence of Wolbachia, a PCR assay using 16S rDNA Wolbachia-specific primers was carried out following the methodology of García-Martínez et al (1998). Six lines were used at random (three of haplotype I and three of haplotype II) from the Calvià population before creating the cages. Since the results were negative, the presence of Wolbachia in the population was excluded. We only used a few lines of D. subobscura because this determination was the second one made on the island of Majorca. The first one was in a different population (Esporles) a few years ago and was also negative (García-Martínez et al, 1998). Moreover, we have not detected any kind of cytoplasmic incompatibility in the population, such as embryonic death or sex ratio distortion in crosses with different isofemale lines.

\section{Experiments}

Egg viability and developmental time: Four groups of approximately 15 adult couples per haplotype were transferred for $24 \mathrm{~h}$ from the $500 \mathrm{ml}$ maintaining bottles to $250 \mathrm{ml}$ bottles with fresh food in order to provide sufficient adult offspring reared without competition. At 2 or 3 days after eclosion, offsprings (males and females together) were transferred to new $250 \mathrm{ml}$ bottles with fresh food, where the flies could mate, feed and lay eggs freely for 1 week. These flies with 9-10 days of age were used for egg laying. They were transferred for $1 \mathrm{~h}$ to 'oviposition vials', which contained a watch glass with agar, water, acetic acid, ethyl alcohol and a few milligrams of live yeast.

The eggs were laid on three watch glasses per haplotype and were immediately ordered into columns. Eggs were checked at $0,14,23,35 \mathrm{~h}$, and then at intervals of $30 \mathrm{~min}$ until $48 \mathrm{~h}$, before being checked the next day at $62 \mathrm{~h}$. Hatching was deduced from the number of empty eggs found. Four replicates were carried out on 4 consecutive days and the number of eggs used in each was $161 / 160,125 / 130,125 / 147$ and $74 / 69$, for haplotypes I and II, respectively. This procedure allowed the variations in developmental time and egg viability of each haplotype to be followed.

Larva-adult viability and developmental time without competition: Once having obtained eggs as in the previous experiment (except that egg laying lasted $2 \mathrm{~h}$ instead of one), they were kept in Petri dishes for $48 \mathrm{~h}$ at $19^{\circ} \mathrm{C}$ until the larvae hatched. Then 50 larvae of the same age were seeded in $10 \times 3 \mathrm{~cm}$ tubes with $10 \mathrm{ml}$ of fresh food. Larvae were picked up one by one directly from the watch glasses with a lancet under a microscope. The adults that emerged were counted daily, and a total of 13 replicates were carried out for each haplotype. 
Viability was expressed as:

$$
V=\frac{n}{N}
$$

where $N$ is the input number of larvae and $n$ is the output number of adults emerging from these $N$ larvae.

Developmental time (DT) was measured in days by the formula:

$$
\mathrm{DT}=\frac{\sum N_{i} d_{i}}{\sum N_{i}}
$$

where $N_{i}$ is the number of flies emerging $d_{i}$ days after the larvae were placed on the medium.

Longevity: The flies tested for longevity were the offspring taken straight from the isofemale line population founder tubes, as they hatched from their pupae. Approximately 30 individuals of each haplotype and sex were maintained individually in $10 \times 3 \mathrm{~cm}$ tubes with $10 \mathrm{ml}$ of food medium containing active yeast on the surface. Flies were checked daily. Vials were changed every fortnight, before dehydration of food was evident. All the flies that escaped, were damaged or died during the experiment were included in the analyses.

Resistance to desiccation: In all, 30 flies of 8 days of age per haplotype and sex were put individually in empty $10 \times 1.1 \mathrm{~cm}$ tubes, and they were checked hourly from the 23rd h until death. Flies for experiments were obtained as above.

Optimal density: Larvae were obtained with the same methodology indicated in the second experiment. Larvae were seeded in $7.5 \times 1 \mathrm{~cm}$ tubes with $0.75 \mathrm{ml}$ of food, in increasing densities of 10, 20, 30, 40, 50, 60, 70, 80, 90 and 100 larvae. Adults emerging at each level were counted daily, and a total of four replicates were carried out for each haplotype.

\section{Statistics}

Statistical analyses were performed by means of the SPSS V 11.5 and the BIOM (Sokal and Rohlf, 1995) packages. Viabilities were subjected to the arcsine-square root transformation for statistical analyses, but the results with transformed data were qualitatively indistinguishable from analyses with untransformed data, so only the latter are presented. The longevity and the resistance to desiccation were analysed by means of the survival analysis procedure (included in the SPSS package). The method was that of Kaplan-Meier survival curves. The analysis gives, among others parameters, the cumulative survival with time and the mean with standard error (SE) for each curve. The comparison of the several curves was made with the log-rank test, so called because it can be shown to be related to a test that uses the logarithms of the ranks of the data. We made comparisons of the four curves globally and by pairs. To diminish any statistical artefacts in the significance that could arise when multiple comparisons are made, a sequential Bonferroni test (Rice, 1989) was applied to correct the probabilities when many tests are carried out simultaneously.

E-statistic (Moya et al, 1986) is a fitness parameter that combines data of viabilities and developmental times, and follows the optimality principle that the largest number of adults emerged in the shortest possible time gives the best fitness. The characteristic of this parameter is that it simultaneously maximizes viability, $V$, and the reciprocal of the development time, DT, as follows:

$$
E_{\max }=\max \left(V \frac{1}{\mathrm{DT}}\right)
$$

The function adopted for $E$ is

$$
E=\sum_{i=0}^{k} \frac{V_{i}}{T_{i}}
$$

where $V_{i}=s_{i} / N$ and $T_{i}=t_{i} / t_{\text {optimal }} ; s_{i}$ is the number of individuals emerged at $t_{i}$ time; $N$ is the input number; and $t_{\text {optimal }}$ is a conventional minimum developmental time (in the egg-larva test it was considered as $40 \mathrm{~h}$, and in the larva-adult test as 20 days).

Hence, for computational purposes we can use

$$
E=\frac{t_{\text {optimal }}}{N} \sum_{i=0}^{k} \frac{s_{i}}{t_{i}}
$$

It is worthy of remark that E-statistic is only a combination of viabilities and developmental times; thus, it does not include other fitness components such as population growth rates or fecundities.

\section{Results}

No differences in the egg-to-larva viability, developmental time or the E-statistic were statistically significant, although the trend was the same as found by Matos and Rocha Pité (1989) for young female flies, where percentages for eclosion showed very high values, close to or attaining $100 \%$. Flies with haplotype II had higher viability and developed faster than haplotype I (Table 1). The same table shows the larva-to-adult viabilities, developmental times and E-statistic, always favouring haplotype II, although statistically different were only found in the DT and E. However, in both experiments, the developmental times were longer than those of previous studies (Rocha Pité, 1982) where this species developed faster even at lower temperatures. In both strains, the viabilities were low and in the same range as those found in the literature (Budnik et al, 1991).

The survival curves for the adult males and females of both haplotypes are shown in Figure 1. As can be observed in Table 2, the females of both haplotypes have similar mean longevity and lived longer than males. The survival analyses showed a great similarity in the dynamics of both females, but not in males, different between them and in relation to the females, as indicated by the log-rank tests (Table 2). Longevity results were similar to those of Maynard Smith (1956) in that many female flies were alive 100 days after eclosion, but they were dissimilar to Clarke and Maynard Smith (1955), whose flies all lived less than 96 days.

Haplotype II was more resistant to desiccation than haplotype I in both sexes, as can be seen in Figure 2 . Within the haplotypes, females were more resistant than their corresponding males. These results are in accordance with those obtained by Levine (1986) for D. pseudoobscura and D. melanogaster. All comparisons were significant except between haplotype II males and both female haplotypes (see Table 2). 
Table 1 Means with SEs for the egg-larva and larva-adult $V$, DT and E-statistic for haplotypes I and II

\begin{tabular}{|c|c|c|c|c|}
\hline & \multicolumn{2}{|c|}{ Haplotypes } & \multirow[t]{2}{*}{$\mathrm{t}$-Test } & \multirow[t]{2}{*}{$d f$} \\
\hline & $I$ & II & & \\
\hline \multicolumn{5}{|l|}{ Egg-larva } \\
\hline$V$ & $0.968 \pm 0.014$ & $0.988 \pm 0.006$ & 1.32 (NS) & 6 \\
\hline DT (hours) & $42.49 \pm 0.18$ & $42.15 \pm 0.40$ & 0.77 (NS) & 6 \\
\hline$E$ & $0.914 \pm 0.016$ & $0.943 \pm 0.012$ & 1.42 (NS) & 6 \\
\hline \multicolumn{5}{|l|}{ Larva-adult } \\
\hline$V$ & $0.632 \pm 0.024$ & $0.689 \pm 0.023$ & 1.73 (NS) & 24 \\
\hline DT (days) & $24.75 \pm 0.20$ & $23.21 \pm 0.08$ & $6.99^{* * *}$ & 24 \\
\hline$E$ & $0.512 \pm 0.017$ & $0.595 \pm 0.019$ & $3.19^{* *}$ & 24 \\
\hline
\end{tabular}

df: degrees of freedom.

NS: not significant.

** $P<0.01$.

$* * * P<0.001$



Figure 1 Survival curves for longevity (in days) for haplotypes I and II (MI and MII: males; FI and FII: females). Censored individuals are indicated by stars.

Table 3 shows the $V$ 's, DTs and E-statistic with SEs for each density and haplotype from the OD test. As a general trend, haplotype II had a higher $V$ but also a higher DT. E-statistic showed globally a higher value for haplotype II. In this experiment, only trends were detected in the results, because, overall, there were no statistically significant differences in the densities between the two haplotypes with respect to the three parameters (haplotype II in $V$ and $E$ were significantly higher than haplotype $I$ in only the 10 and 60 density bands). It is possible that more replicates in this experiment would confirm these tendencies. Nevertheless, these results show that the differences between the two haplotypes were very small.

As expected, viability and developmental time were density-dependent parameters, with the first decreasing and the second increasing as the density increased. In the literature, it is reported that the best statistical fit of these parameters is not always linear and it is common to fit the data to a second- or third-degree polynomial regression (see, for example, Castro et al, 1985 and references inside for a detailed explanation of the method). Table 4 shows the best fit of the $V^{\prime} s$, DTs and $E$ by density that corresponded to second-degree polynomial regressions based on the analysis of variance for both haplotypes. From the polynomic regression, optimal densities of 32 individuals for haplotypes I and II were obtained in viability, but haplotype II showed a slightly better use of the medium by having a higher number of survivors at this OD (11.7 vs 9.6). The results with $E$ were similar (Table 4 and Figure 3$)$. The curves in Figure 3 resulted from the polynomic regression for $V$ and $E$ multiplied by $N$. Only the first part of the curves (up to density 50) that include the OD values are represented, and the original survival points are also indicated. This methodology coincides with similar 
experiments on D. melanogaster. The crowding conditions caused by progressive density provoke an increasing exploitation of the medium to a maximum level (the OD). From this point onwards, the limitation of resources and the increasing concentration of nitrogen catabolites from larval excretion cause a self-poisoning of the larvae, which explain, at least in part, the dynamics of the process, mainly at high densities (Castro et al, 1985; Moya and Botella, 1985; Moya and Castro, 1986; Botella et al, 1988)

Table 2 Means with SEs for longevity (in days) and desiccation resistance (in h) for sexes in haplotypes I and II. The log-rank tests based on the survival analyses are indicated (the global analysis and by pairs)

\begin{tabular}{|c|c|c|}
\hline & Longevity & $\begin{array}{c}\text { Desiccation } \\
\text { resistance }\end{array}$ \\
\hline MI & $65.14 \pm 3.88$ & $35.57 \pm 0.95$ \\
\hline MII & $76.54 \pm 5.25$ & $42.20 \pm 2.12$ \\
\hline FI & $93.48 \pm 5.27$ & $39.55 \pm 1.07$ \\
\hline FII & $94.74 \pm 5.54$ & $46.27 \pm 2.05$ \\
\hline Global & $39.20^{* * *} \quad(3)$ & $28.44^{* * *}$ \\
\hline MI-MII & $8.00^{*} \quad(1)$ & $10.46^{* *} \quad(1)$ \\
\hline FI-FII & 0.06 (NS) (1) & $9.16^{* *} \quad(1)$ \\
\hline MI-FI & $27.54^{* * *}$ & $6.32^{*} \quad(1)$ \\
\hline MII-FII & $6.59^{*} \quad(1)$ & 1.38 (NS) (1) \\
\hline MI-FII & $21.86^{* * *} \quad(1)$ & $22.36^{* * *} \quad(1)$ \\
\hline MII-FI & $9.38^{* *} \quad(1)$ & 3.38 (NS) (1) \\
\hline
\end{tabular}

MI and FI: males and females for haplotype I.

MII and FII: males and females for haplotype II.

NS: not significant.

${ }^{*} P<0.05$.

$* * P<0.01$.

$* * * P<0.001$.

The probabilities were corrected by the sequential Bonferroni test.

Degrees of freedom are given within parentheses.

\section{Discussion}

We have tested the differences in some fitness and lifehistory components for the two main RFLP haplotypes, I and II, in an attempt to find differences that would help us to understand their populational dynamics. We have studied those characteristics commonly used in the literature (Hedrick, 2000), although we are conscious that the prediction of the outcome of natural selection based on separate fitness components could be risky (Prout, 1971a, b). As a general trend, it was observed that haplotype II was more efficient than haplotype I.

Egg viabilities, egg-larva developmental times and Estatistic gave no significant differences. The larva-adult developmental time was significantly shorter for haplotype II and the global $E$-statistic was higher. These results indicate an advantage over haplotype I because in population cages, with discrete generations, we can follow the optimality principle that the largest number of adults emerging in the shortest possible time gives the best fitness. In these laboratory conditions, flies that emerge earlier can mate and lay eggs earlier and, as a consequence, their larvae take the best place on the medium and so feed better. However, this explanation cannot be fully applied to natural conditions, because trade-offs may exist in such a way that slower could be better under certain conditions due to the complexity existing in nature. In relation to $E$-statistic, we think that it is a good approximation to the global fitness of viability and developmental time, although we are aware that the developmental time could have an exponential impact on reproduction, whereas the other traits (not just viability) may only be multiplicative.

For longevity, haplotype II showed a higher longevity trend in both sexes, although significant differences within sexes were found only in males. This advantage is relevant in females because the mtDNA has a maternal transmission, whereas it is not relevant in males because



Figure 2 Survival curves for the desiccation resistance (in hours) for males and females of haplotypes I and II. 
Table 3 Mean $V^{\prime}$ s, DTs (in days) and E-statistic with SEs for each density and haplotype

\begin{tabular}{|c|c|c|c|c|c|c|}
\hline \multirow[t]{2}{*}{ Larval density } & \multicolumn{2}{|c|}{ V } & \multicolumn{2}{|c|}{$D T$} & \multicolumn{2}{|c|}{$\mathrm{E}$} \\
\hline & $I$ & II & $I$ & $I I$ & $I$ & II \\
\hline 10 & $0.52 \pm 0.07$ & $0.75 \pm 0.03$ & $22.72 \pm 0.25$ & $23.81 \pm 0.43$ & $0.461 \pm 0.062$ & $0.632 \pm 0.014$ \\
\hline 20 & $0.42 \pm 0.04$ & $0.44 \pm 0.02$ & $25.46 \pm 0.79$ & $28.02 \pm 0.99$ & $0.334 \pm 0.027$ & $0.317 \pm 0.029$ \\
\hline 30 & $0.36 \pm 0.07$ & $0.31 \pm 0.04$ & $30.93 \pm 0.83$ & $34.27 \pm 1.17$ & $0.233 \pm 0.045$ & $0.188 \pm 0.027$ \\
\hline 40 & $0.22 \pm 0.05$ & $0.26 \pm 0.04$ & $36.41 \pm 0.86$ & $36.53 \pm 1.04$ & $0.127 \pm 0.029$ & $0.144 \pm 0.026$ \\
\hline 50 & $0.14 \pm 0.02$ & $0.16 \pm 0.03$ & $43.00 \pm 2.29$ & $42.84 \pm 1.54$ & $0.072 \pm 0.014$ & $0.081 \pm 0.016$ \\
\hline 60 & $0.06 \pm 0.02$ & $0.16 \pm 0.02$ & $45.36 \pm 2.85$ & $44.75 \pm 1.94$ & $0.027 \pm 0.008$ & $0.078 \pm 0.009$ \\
\hline 70 & $0.07 \pm 0.01$ & $0.11 \pm 0.02$ & $47.08 \pm 2.04$ & $45.92 \pm 3.72$ & $0.033 \pm 0.006$ & $0.050 \pm 0.007$ \\
\hline 80 & $0.05 \pm 0.01$ & $0.06 \pm 0.01$ & $47.87 \pm 3.29$ & $53.01 \pm 1.35$ & $0.023 \pm 0.008$ & $0.024 \pm 0.005$ \\
\hline 90 & $0.06 \pm 0.02$ & $0.08 \pm 0.01$ & $45.46 \pm 4.24$ & $50.61 \pm 1.26$ & $0.026 \pm 0.010$ & $0.033 \pm 0.006$ \\
\hline 100 & $0.06 \pm 0.02$ & $0.07 \pm 0.01$ & $51.16 \pm 3.08$ & $54.20 \pm 1.11$ & $0.026 \pm 0.012$ & $0.029 \pm 0.002$ \\
\hline
\end{tabular}

Table 4 Parameter values for the polynomial regression and OD (with the corresponding survival within parentheses) for each of the three fitness components in the two haplotypes

\begin{tabular}{|c|c|c|c|c|c|c|}
\hline \multirow{2}{*}{$\begin{array}{l}\text { Fitness } \\
\text { Components }\end{array}$} & \multicolumn{3}{|c|}{ Haplotype I } & \multicolumn{3}{|c|}{ Haplotype II } \\
\hline & Parameter values & $\mathrm{R}^{2}$ & $O D(S)$ & Parameter values & $\mathrm{R}^{2}$ & $O D(S)$ \\
\hline$V$ & $\begin{aligned} a & =0.689 \\
b_{1} & =-1.5 \mathrm{E}-2 \\
b_{2} & =9 \mathrm{E}-5\end{aligned}$ & 0.984 & $32(9.6)$ & $\begin{aligned} a & =0.850 \\
b_{1} & =-1.90 \mathrm{E}-2 \\
b_{2} & =1.20 \mathrm{E}-4\end{aligned}$ & 0.951 & $32(11.7)$ \\
\hline DT & $\begin{aligned} a & =14.25 \\
b_{1} & =0.719 \\
b_{2} & =-3.70 \mathrm{E}-3\end{aligned}$ & 0.966 & & $\begin{aligned} a & =17.97 \\
b_{1} & =0.583 \\
b_{2} & =-2.25 \mathrm{E}-3\end{aligned}$ & 0.983 & \\
\hline E & $\begin{array}{c}a=0.594 \\
b_{1}=-0.015 \\
b_{2}=9.5 \mathrm{E}-5\end{array}$ & 0.990 & $26(7.0)$ & $\begin{aligned} a & =0.712 \\
b_{1} & =-0.019 \\
b_{2} & =1.23 \mathrm{E}-4\end{aligned}$ & 0.921 & $25(8.0)$ \\
\hline
\end{tabular}

$R^{2}$ measures the proportion of the experimental variance explained by the regression.

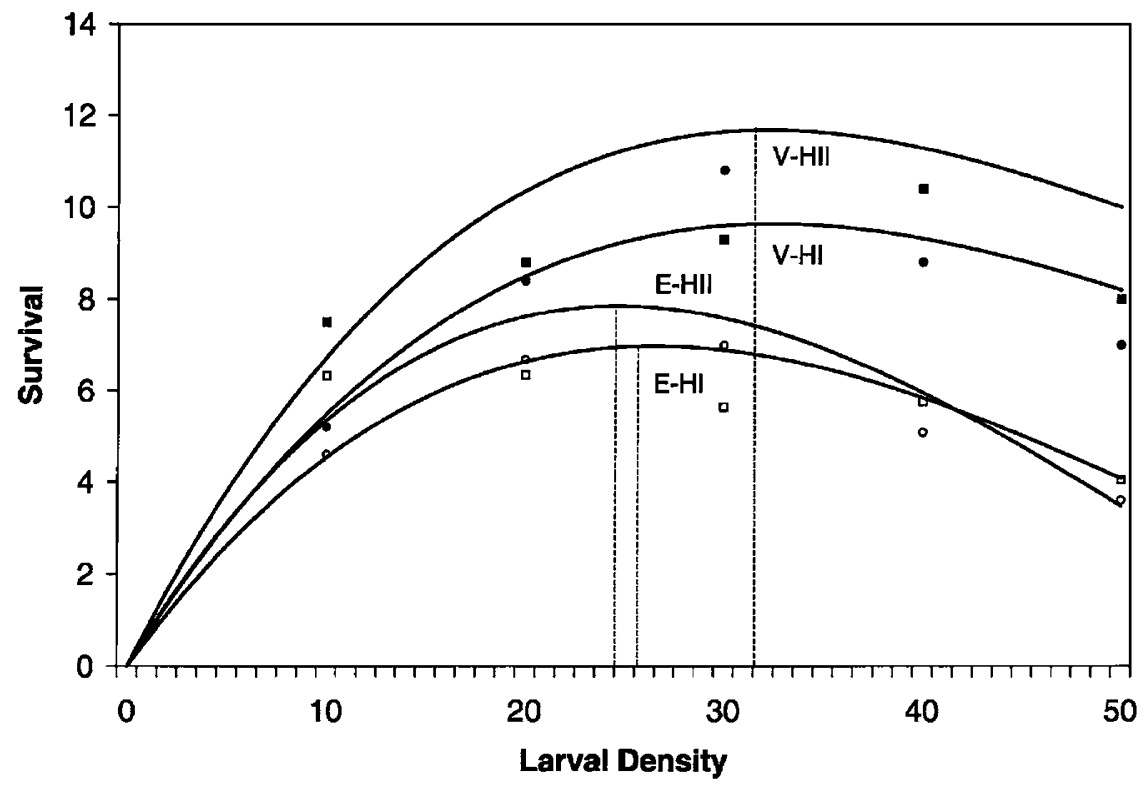

Figure 3 Viability $(V)$ and E-statistic $(E)$ as a function of the input densities for the two haplotypes I and II. The predicted function is shown. The vertical dash lines indicate the optimal input density. The original survival points are also represented (VHI: $\bullet$; VHII: $\mathbf{\square}$; EHI: $\bigcirc$; EHII: $\square$ ).

they do not transfer it. Nevertheless, males contribute with nuclear genes, which generates the nuclear variability necessary in cytonuclear relationships. In this sense, as indicated above, we also consider the mating pattern relevant, and this is an experiment that we carried out (Castro et al, 2003); in this experiment, the mating pattern indicated an assortative mating in population cages, where couples of the same haplotype 
mated more often. Nevertheless, in wild populations, random mating was the rule.

In resistance to desiccation, both males and females of haplotype II are superior to haplotype I. Ecologically, this would mean a higher fitness in haplotype II flies during the driest periods of the year. This is supported by González et al (1994) whose autumn samples (flies and descendants of flies just after the dry season) showed larger frequency differences between the two main haplotypes in favour of haplotype II compared to their spring samples. The cause of a difference between haplotypes and not just sexes could be explained by differences in certain proteins that would result in a different functionality with conditions of rising cell osmolarity during the dehydration of the fly. Interestingly, Kennington et al (2001) showed, by means of this trait, the importance of the maternal contribution to population differentiation in Drosophila melanogaster.

In the OD experiment, the general trend was for haplotype II to make the best use of the medium, since the survival of haplotype II at OD (which has some similarities with carrying capacity in population dynamics studies) was higher than that of haplotype I. This means that haplotype II has a better fitness than haplotype I so that it can profit from the laboratory medium, and it is possible that this advantage persists in nature when seasonal conditions are extreme. However, the interpolation from monocultures to dicultures must be treated with caution, because the response pattern of both haplotypes competing together is not fully predictable from monocultures due to intergenotypic competition (Castro et al, 1985).

The differences in fitness component values found in the present work raise certain questions. (1) What are these fitness components measuring? We think that selection is acting differentially on the two haplotypes, either directly on the mtDNA or by some kind of selective coadaptation between the nuclear and the mtDNA genomes (epistatic selection and/or hitchhiking on mtDNA haplotypes). At a nuclear level, we can reasonably assume that both haplotypes had the same nuclear variability when the populational cages were founded, because of the high number of isofemale lines used for each haplotype. At the mtDNA level, 15\% of the sequence of the mtDNA molecule showed three silent changes, including that of HaeIII. It is expected (and is probably true) that more differences could be found (not necessarily silent) in the remaining $85 \%$. With our experimental design, we cannot distinguish clearly between factors that are exclusively from mtDNA for nuclear-mtDNA interactions, although we can say that the mtDNA is always implicated. Previous data (Castro et al, 1999; Oliver et al, 2002) showed the presence of cytonuclear disequilibria, permitting the possibility of some kind of epistatic selection. Despite the transience of the cytonuclear interactions (Babcock and Asmussen, 1996), in the cages and after nine generations, it is possible that some could be formed (differently in both cages) in response to laboratory environmental homogeneity, and therefore persist over time (or reappear recurrently). The consequence would be a net superiority of haplotype II over haplotype I, because the former could have developed a better functional coadaptation than the latter, as also indicated by Fos et al (1990). This would be mainly in the nuclear genes related to the more relevant fitness components (resistance to desiccation, longevity and optimum density). (2) Can these differences be extrapolated to nature? The life in a population cage is very different from nature (no microhabitats, no differences in food, the same temperature and humidity, the nonexistence of early morning dew, a closed environment with competition for food and space, etc.), so a direct extrapolation to nature is not possible. In fact, Castro et al (2003) found, in relation to female fertility, that the adaptation to laboratory conditions gave a more global efficiency in the production of the offspring. In this situation, we can only say that under laboratory conditions, flies with haplotype II have a higher fitness than flies with haplotype I, and, thus, haplotype II would displace haplotype I in competition experiments. This was exactly what García-Martínez et al (1998) found in population cages. Therefore, our experiments could explain, at least partially, the displacement of haplotype I by haplotype II in those experiments. In nature, the situation is more complicated than in laboratory cages, and the superiority of one haplotype must be counterbalanced by the other in different situations. The demographic structural characteristics in D. subobscura have shown significant differences between seasons (Matos and Rocha Pité, 1989). Since patterns differ remarkably throughout the year, a seasonal analysis could be important to characterize the demographic parameters of each haplotype, as one haplotype is favoured momentarily to the detriment to the other. Therefore, flies with haplotype I must have some advantage over flies with haplotype II in order to explain the proportions found in nature, developing cytonuclear interactions, even transient, that could have selective importance in a changing environment.

Finally, we have to consider the sex-by-genotype interactions because they could, in part, maintain the variation in the populations. In this way, Rand et al (2001) developed a model for $D$. melanogaster in which nuclearcytoplasmic polymorphism could be maintained by selection in X chromosome-cytoplasm interactions. They showed experimentally significant sex-by-genotype interactions for mtDNA haplotype, cytoplasms (the factors inherited through the female cytoplasm in Drosophila with different mtDNA, such as Wolbachia, $\sigma$ and $C$ virus and maternally loaded mRNA) and $X$ chromosomes. Moreover, these interactions were sexually antagonistic (the good cytoplasm in females was bad in males). This model reveals the complexity of the interactions that we have to take into account.

\section{Acknowledgements}

This work has been supported by Grant PB96-0793 and BOS2000-1000 from the Dirección General de Enseñanza Superior (Ministerio de Educación y Cultura, Spain).

\section{References}

Babcock CS, Asmussen M (1996). Effects of differential selection in the sexes on cytonuclear polymorphism and disequilibria. Genetics 144: 839-853.

Botella LM, Moya A, Mensua JL (1988). The effects of competition for food on pupal mortality in Drosophila. Evol Biol 2: 109-121. 
Budnik M, Cifuentes L, Brncic O (1991). Quantitative analysis of genetic differentiation among European and Chilean strains of Drosophila subobscura. Heredity 67: 29-33.

Castro J, Moya A, Mensua JL (1985). Competitive selection in mono-, di-, and tri-genotype cultures of Drosophila melanogaster. Z Zool Syst Evolut-forsch 23: 214-228.

Castro JA, Oliver P, Christie JS, Picornell A, Ramon M, Moya A (2003). Assortative mating and fertility in two Drosophila subobscura strains with different mitochondrial DNA haplotypes. Genetica 119: 295-301.

Castro JA, Ramon M, Picornell A, Moya A (1999). The genetic structure of Drosophila suboscura populations from the islands of Majorca and Minorca (Balearic Islands, Spain) based on allozymes and mitochondrial DNA. Heredity 83: 271-279.

Clark AG, Lyckegaard EMS (1988). Natural selection with nuclear and cytoplasmatic transmission. III. Joint analysis of segregation and mtDNA in Drosophila melanogaster. Genetics 118: 471-481.

Clarke JM, Maynard Smith J (1955). The genetics and cytology of Drosophila suboscura. XI. Hybrid vigour and longevity. J Genet 53: 172.

Fos M, Domínguez MA, Latorre A, Moya A (1990). Mitochondrial DNA evolution in experimental populations of Drosophila subobscura. Proc Natl Acad Sci USA 87: 4198-4201.

García-Martínez J, Castro JA, Ramón M, Latorre A, Moya A (1998). Mitochondrial DNA haplotype frequencies in natural and experimental populations of Drosophila subobscura. Genetics 149: 1377-1382.

Gerber AS, Loggins R, Kumar S, Dowling TE (2001). Does nonneutral evolution shape observed patterns of DNA variation in animal mitochondrial genomes? Ann Rev Genet 35: $539-566$.

González A, Carrió R, Fernández-Pedrosa V, Moya A (1994). Lack of seasonal changes in mitochondrial DNA variability of a Drosophila subobscura population. J Evol Biol 7: 29-38.

Hedrick PW (2000). Genetics of Populations, 2nd edn. Jones and Barlett Publishers International: London.

Huey RBL, Partridge L, Fowler K (1991). Thermal sensitivity of Drosophila melanogaster responds rapidly to laboratory natural selection. Evolution 45: 751-756.

Hutter CM, Rand DM (1995). Competition between mitochondrial haplotypes in distinct nuclear genetic environments: Drosophila pseudoobscura vs D. persimilis. Genetics 140: 537-548.

Kennington WJ, Gilchrist AS, Goldstein DB, Partridge L (2001). The genetic bases of divergence in desiccation and starvation resistance among tropical and temperate populations of Drosophila melanogaster. Heredity 87: 363-372.

Latorre A, Hernández C, Martínez D, Castro JA, Ramón M, Moya A (1992). Population structure and mitochondrial DNA gene flow in Old World populations of Drosophila suboscura. Heredity 68: 15-24.

Levine L (1986). Desiccation resistance in two species of Drosophila. J Hered 77: 359-360.

MacRae AF, Anderson WW (1988). Evidence for non-neutrality of mitochondrial DNA haplotypes in Drosophila pseudobscura. Genetics 120: 485-494.

Matos M, Rocha Pité MT (1989). Egg viability in Drosophila subobscura throughout one year under semi-natural conditions. Evol Biol 3: 369-382.
Matos M, Rose MR, Rocha Pité MT, Rego C, Avelar T (2000). Adaptation to the laboratory environment in Drosophila subobscura. J Evol Biol 13: 9-19.

Maynard Smith J (1956). Fertility, mating behaviour and sexual selection in Drosophila suboscura. J Genet 54: 261-279.

Moya A, Barrio E, Martínez D, Latorre A, González-Candelas F, Ramón M et al (1993). Molecular characterization and cytonuclear disequilibria of two Drosophila subobscura mitochondrial haplotypes. Genome 36: 890-898.

Moya A, Botella LM (1985). Larva-to-adult and pupa-to-adult mortality dynamics in crowded cultures of Drosophila melanogaster. Genetica 67: 201-207.

Moya A, Castro JA (1986). Larval competition in Drosophila melanogaster: the model of the bands of density. Oikos 47: 280-286.

Moya A, González-Candelas F, Ayala FJ (1986). Intra- and intergenotypic competition in Drosophila melanogaster: effects of density on larval survival and rate of development. Genetica 70: 59-67.

Mueller LD, Graves JL, Rose MR (1993). Interactions between density-dependent and age-specific selection in Drosophila melanogaster. Funct Ecol 7: 469-479.

Oliver P, Castro JA, Picornell A, Ramon MM, Solé E, Balanyà J et al (2002). Linkage disequilibria between mtDNA haplotypes and chromosomal arrangements in a natural population of Drosophila subobscura. Heredity 89: 133-138.

Pinto FM, Brehm A, Hernandez M, Larruga JM, González AM, Cabrera VM (1997). Population genetic structure and colonization sequence of Drosophila subobscura in the Canaries and Madeira Atlantic islands as inferred by autosomal, sex-linked and mtDNA traits. J Hered 88: 108-114.

Prout T (1971a). The relation between fitness components and population prediction in Drosophila. I: the estimation of fitness components. Genetics 68: 127-149.

Prout T (1971b). The relation between fitness components and population prediction in Drosophila. II: population prediction. Genetics 68: 151-167.

Rand DM, Clark AG, Kann LM (2001). Sexually antagonistic cytonuclear fitness interaction in Drosophila melanogaster. Genetics 159: 173-187.

Rice WR (1989). Analyzing tables of statistical tests. Evolution 43: 223-225.

Rocha Pité MT (1982). Influence of temperature on physiological characters of D. phalerata, D. suboscura and D. simulans (Insecta, Diptera, Drosophilidae.). 1 - Larval-pupal mortality and duration of the development. Bolm Soc Port Ent 27: 1-16.

Rose MR (1984). Laboratory evolution of postponed senescence in Drosophila melanogaster. Evolution 38: 1004-1010.

Rose MR, Vu LN, Park SU, Graves JL (1992). Selection on stress resistance increases longevity in Drosophila melanogaster. Exp Gerontol 27: 241-250.

Rozas JM, Hernandez M, Cabrera VM, Prevosti A (1990). Colonization of America by Drosophila subobscura: effect of the founder event on the mitochondrial DNA polymorphism. Mol Biol Evol 7: 103-109.

Singh RS, Hale LR (1990). Are mitochondrial DNA variants selectively non-neutral? Genetics 124: 995-997.

Sokal RR, Rohlf FJ (1995). Biometry, 3rd edn. WH Freeman and Co: New York.

Wallace B (1981). Basic Population Genetics. Columbia University Press: New York. 